Bayesian Exchangeability, Benefit Transfer, and Research Efficiency

Scott E. Atkinson Thomas D. Crocker

Jason F. Shogren

1992

Journal Article WWRC-92-04

In

Water Resources Research

Volume 28

Submitted by

Scott E. Atkinson

Department of Economics

University of Georgia

Athens, Georgia

Thomas D. Crocker

Department of Economics

University of Wyoming

Laramie, Wyoming

and

Jason F. Shogren

Department of Economics

Iowa State University

Ames, Iowa 


\title{
Bayesian Exchangeability, Benefit Transfer, and Research Efficiency
}

\author{
SCOTt E. AtKInSON \\ Department of Economics, University of Georgia, Athens \\ Thomas D. Crocker \\ Department of Economics and Finance, University of Wyoming, Laramie
}

JASON F. SHOGREN

Department of Economics, Iowa State University, Ames

\begin{abstract}
We offer an economic model of the policymaker's site- or time-specific benefit estimate extrapolation problem when she must weigh the potential gains from an increase in the accuracy and the precision of her agents' estimates against the costs of conducting their assessments. If Bayesian exchangeability is treated as a maintained hypothesis, we suggest that empirical Bayes estimators offer a powerful way to increase the economic efficiency of extrapolation. Finally, we employ a hedonic study of pollution control benefits to illustrate a Bayesian diagnostic that allows the hypothesis of exchangeability to be tested rather than taken as maintained. The power of the diagnostic arises from its ability to identify those sources of parameter variability most likely to discourage extrapolations.
\end{abstract}

\section{INTRODUCTION}

Public and private agencies expend considerable resources to quantify the economic consequences of altering service flows and stocks of nonmarketed features of the natural environment, including those involving water. In substantial part, former President Reagan's Executive Order 12291 and assorted legislative and juridical mandates motivate acquisition of this information. Nevertheless, limited research budgets and immediate demands to "do something" beyond studying a problem inhibit a proliferation of valuation studies unique to each site, time, and resource allocation system. When confronted with a demand for yet another site-specific environmental improvement, policymakers must decide whether to extrapolate the results of benefit assessments done elsewhere or to commission a new assessment study. This paper suggests practical means for evaluating the extent of the information increase extrapolation might provide. We propose means whereby policymakers can assess how much is to be learned from other benefit studies. Our proposal rests upon the Bayesian concept of exchangeability.

As originally set forth by de Finetti [1972], exchangeability presumes the existence of a grand model which generates random samples in a number of distinct groups; the individual groups are a priori thought to possess characteristics sufficiently similar that a common structure applies to each. Each group represents an imperfect replication of the common structure. Given exchangeability, Bayesian estimators allow one to draw systematic and communicable inferences about a parameter value in one group from the observations on all groups. If exchangeability is complete the identical model would apply to each group. One could then pool the data from all studies and transfer without revision the results

Copyright 1992 by the American Geophysical Union.

Paper number 91WR02598.

0043-1397/92/91WR-02598\$05.00 for one group to any other group. At the other extreme, if exchangeability is utterly absent, then each group has its own unique structure and no transfer of results or pooling of data would be justified. Each group would be totally idiosyncratic.

Between these two extremes the Lindley and Smith [1972] formulation of exchangeability produces a weighted average of the data from other samples and data from the group (time, place, and allocation process) of concern, where the precision of the pooled versus the individual sample estimates determines the weights. Rather than assuming that the benefits of some policy change are either totally similar or dissimilar across times, locations, or allocation systems, one can evaluate the extent to which they are similar. For many policy problems thebenefit estimates from one setting, though not derived from a structure identical to that in the setting of immediate concern, may nevertheless be close enough to discriminate adequately among relevant policy alternatives. The practical implementation of exchangeability concepts allows the policymaker to assess the degree of closeness among groups as well as the implications for estimating payoffs of policy alternatives [e.g., Adams et al., 1984]. It recognizes that a central aim of inference is to generate predictions about the values of distant observables.

The few existing systematic efforts to extract commonality from ensembles of water resource benefit studies [e.g., Walsh et al., 1989; Smith and Kaoru, 1990] implicitly treat exchangeability as a maintained hypothesis. These investigations examine how benefit estimates differ with the fixed effects of previous studies' settings, restrictions, and methods. However, because they assume all of the variance among study fixed effects other than the sampling variance can be deterministically explained as a function of known study characteristics, their applications to the benefit transfer problem may be unnecessarily restrictive. When one does not account for stochastic sources of variation, the estimated expression is misspecified, meaning that the fixed 
parameter estimates and standard errors are untrustworthy. Chow's [1981] and similar homogeneity tests for fixed effect size might be used to detect model misspecification, but when no specification survives these tests, it is unclear how one might proceed.

If exchangeability is treated as a maintained hypothesis, empirical Bayes estimators that treat some or all individual effects as random offer a powerful way to proceed. In the next section, we offer an economic model that specifies properties of the policymaker's extrapolation problem. Section 3 briefly outlines well-known empirical Bayes estimators that increase the economic efficiency of extrapolation. Section 4 employs a hedonic study of pollution control benefits to illustrate how Bayesian diagnostics allow the hypothesis of exchangeability to be tested rather than taken as maintained. Though the specific application involves air pollution, the identical procedures could be applied to the ensemble of water recreation benefits studies used by Walsh et al. [1989] and Smith and Kaoru [1990].

\section{The Problem of Efficient Benefit Transfers}

The above discussion describes a problem in optimal information search. Assume that the policymaker's welfare is proportional to the accuracy and the precision of the benefit estimates that her agents provide. She will then collect all benefit information that she can collect, given that this information is costless to acquire. However, benefit assessments are costly. The policymaker must therefore weigh the potential benefits from an increase in the accuracy and the precision of her agents' estimates against the costs of conducting their assessments [Crocker, 1975].

Consider an individual whom the policymaker views as representative and who acquires weakly separable utility from frequenting a finite number of recreational sites. Presume that policymaker agents have a set of tools which enable them to translate this utility into income equivalents that measure economic benefits. The policymaker wishes to know the benefits that have accrued or would accrue to the ndividual from some perturbation at one or more of the irequented sites.

Define recreational benefits for individual $j=1, \cdots, m$, It site $i=1, \cdots, n$, as $y_{i j}$. We explain $y_{i j}$ using the ittributes, $z_{i j k}$, for each of $k$ individual and site-specific attributes. In addition, three random components may affect $y_{i j}$ :

$$
y_{i j}=\sum_{k} z_{i j k} \beta_{k}+\alpha_{j}+\gamma_{i}+\varepsilon_{i j} \text {, }
$$

vhere

$z_{i j k}$ observation on the $k$ th nonstochastic explanatory variable for the $j$ th individual at the $i$ th site;

$\beta_{k} \quad k$ th population parameter;

$\alpha_{j}$ random component specific to the $j$ th individual or group of individuals;

$\gamma_{i}$ random component specific to the $i$ th site or group of sites;

$\varepsilon_{i j}$ random component specific to the $i$ th site and the $j$ th individual.

We assume that $\varepsilon_{i j}$ is independently and identically disibuted (iid) normal with zero mean and variance $\sigma_{\varepsilon}^{2}$. Further $\alpha_{j}$ and $\gamma_{i}$ are iid normal with zero means and rariances $\sigma_{\alpha}^{2}$ and $\sigma_{\gamma}^{2}$.
Expression (1) says that the recreation sites produce three types of random benefits to the individual. The random component, $\alpha_{j}$, of recreational activities reflects the utility that he obtains from "not working." Factors such as his inherent work ethic, the identities of those who accompany him in any leisure activity, and the state of the overall economy are the sources of this component. This component is common to all recreational sites. The second component, $\gamma_{i}$, is site-specific and is due to random variation in weather patterns, congestion, and animal populations. A third component, $\varepsilon_{i j}$, represents effects that are random across sites and individuals. For example, the individual may better enjoy the happenstance of sunny weather at a site if he knows that his job prospects have been improved by a strong overall economy.

For analytical tractability, we assume that the $z$ components affect every individual similarly. This implies that $\sigma_{\alpha}^{2}$ $=0$, thus allowing us to disregard the $\alpha_{j}$. We also assume that $\gamma$ is a fixed rather than a random effect, and that the only fixed effects are site-specific dummy variables. Expression (1) then becomes a model of the mean where

$$
y_{i j}=\sum_{1} z_{i j} \beta_{i}+\varepsilon_{i j}
$$

where $z_{i j}=1$ if individual $j$ is at any site $i$, otherwise $z_{i j}=$ 0 .

We describe the policymaker's prior belief about $\beta$ with a normal probability density function (pdf) having mean $\mu$ and known variance $\sigma_{\beta}^{2}$. Thus

$$
\beta \sim N\left(\mu, \sigma_{\beta}^{2}\right)
$$

The policymaker uses each new benefits study to revise her prior beliefs about the factors that influence the individual's benefits. We can therefore use Bayes's theorem to obtain a posterior distribution for $\beta$. Following Judge et al. [1988], this posterior pdf will have mean

$$
E\left(\beta_{i} \mid y\right)=\left[1-\frac{\sigma_{\varepsilon}^{2}}{\Delta}\right] \bar{y}_{i}+\left[\frac{\sigma_{\varepsilon}^{2}}{\Delta}\right] \mu
$$

and variance

$$
\operatorname{Var}\left(\beta_{i} \mid y\right)=\frac{\sigma_{\varepsilon}^{2} \sigma_{\beta}^{2}}{\Delta}
$$

where $n$ is the number of study sites, and $\Delta=\sigma_{\varepsilon}^{2}+n \sigma_{\beta}^{2}$.

Expression ( $4 a$ ) says that the policymaker's posterior benefit estimate will be a weighted average of the sample mean, $\bar{y}_{i}$, and her prior belief, $\mu$, where the weights reflect the relative precision of each mean value.

Now consider the policymaker's problem with respect to commissioning additional benefit studies. Her focus is upon management, not inference [Klein et al., 1978]. Accordingly, assume that her loss function, $W$, is a simple linear combination of the variance in expression $(4 b)$ of her posterior pdf and the costs of doing additional benefits studies. Her problem is then

$$
\underset{n}{\operatorname{Min}} W=p[\operatorname{Var}(\beta \mid y)]+g C(n),
$$

where $p$ and $g$ are the implicit pecuniary weights she attaches to greater uncertainty about benefits and to the 
costs of doing benefit studies, and where $C(n)$ is strictly convex increasing. Included in $C(n)$ are the opportunity costs of incorrect specification of a parameter value. The first-order optimality condition for $(5)$ is

$$
-p \sigma_{\varepsilon}^{2}\left(\sigma_{\beta}^{2}\right)^{2} / \Delta^{2}=g C^{\prime}
$$

The second-order condition

$$
D=\left(2 p \sigma_{\varepsilon}^{2}\left(\sigma_{\beta}^{2}\right)^{3} / \Delta^{3}\right)+g C^{\prime \prime}>0
$$

is assumed to hold whenever (6) holds.

Expression (6) implies that an increase in the marginal cost of commissioning studies causes the policymaker to tolerate greater uncertainty about the common benefit and about site-specific benefit information. She will consequently commission fewer site-specific benefit studies. Note that as her uncertainty about common benefits becomes relatively large, she becomes increasingly reluctant to extrapolate the benefit estimates of previous studies to new settings. This result accords with a standard conclusion in the Bayesian econometric literature. The following observations summarize our results.

Observation 1. A policymaker's willingness to transfer benefit estimates to a new site will unambiguously increase if (1) the price of reducing uncertainty about common benefits decreases; (2) the cost of doing a new benefit study increases; and (3) the variance of the prior distribution of common benefits decreases; that is,

$$
\begin{gathered}
\frac{\partial n}{\partial p}=\frac{\sigma_{\varepsilon}^{2}\left(\sigma_{\beta}^{2}\right)^{2}}{D \Delta^{2}}>0, \\
\frac{\partial n}{\partial g}=\frac{-C^{\prime \prime}}{D}<0, \\
\frac{\partial n}{\partial \sigma_{\beta}^{2}}=\frac{2 p\left(\sigma_{\varepsilon}^{2}\right)^{2} \sigma_{\beta}^{2}}{D \Delta^{3}}>0 .
\end{gathered}
$$

Note, however, that the policymaker's incentive to commission additional studies is more complicated with a decrease in the variance of site-specific benefits. Now she must weigh her uncertainty about the common benefit against her average uncertainty about site-specific benefit estimation.

Observation 2. Since

$$
\frac{\partial n}{\partial \sigma_{\varepsilon}^{2}}=\frac{p\left(\sigma_{\beta}^{2}\right)^{2} n}{D \Delta^{3}}\left(\sigma_{\beta}^{2}-\frac{\sigma_{\varepsilon}^{2}}{n}\right),
$$

a decrease in the variance of site-specific benefit information will increase a policymaker's willingness to transfer benefit estimates to a new site unless the variance $\sigma_{\beta}^{2}$ of the common benefit estimate is small relative to the average variance $\left(\sigma_{\varepsilon}^{2} / n\right)$ of the site-specific benefit estimates. Thus an increase in the precision of site-specific benefit estimation may make the policymaker more skeptical about gains to be had from transferring benefit estimates.

\section{Exploiting the Common Benefit}

Assume that the conditions in observations 1 and 2 hold such that the policymaker is strongly tempted to extrapolate some average of the benefit estimates of previous studies to a new setting. Given this temptation, the question remains as to how she might exploit the commonality feature to increase the likelihood of minimizing her expected sum of prediction error losses and study-commissioning costs set forth in (5). A class of statistical methods generally known as random coefficient estimators [e.g., Swamy, 1970] allows her to do so.

Random coefficient approaches are fully consistent with the treatment in (1)-(11) of the parameters of the common benefit as random variables. First, as already noted, benefit influences common to all sites may nevertheless have varying impacts across sites because of differences in the behavior of stochastic influences like weather. Second, a great deal of variation in the common benefit parameters may be expected, not only because of obvious differences in stochastic influences across sites, but because of a variety of other differences much more difficult to isolate. The explanatory value of a particular parameter is readily vitiated when a system involves a large number of unacknowledged parameters. Third, statistical practitioners make it a standard practice to use random variability (disturbances or errors) to "explain" variations that are not explicitly modeled. Fourth, when one works with incompletely specified models, one must inevitably confront the possibility of bias and the loss of efficiency in estimates. Finally, benefit measures are usually derived from statistically estimated demand functions and must therefore be random variables [Just et al., 1982 , p. 76]. In general, economically relevant data for the many nonmarketed goods such as clean water can reasonably be said to have been generated by a random coefficient process. It follows that statistical inference for these settings is appropriately based on a similar story.

More arguments for random coefficient models are readily added to this list. Random coefficient models are more inclusive because fixed coefficients are simply a special case of a random variable [Swamy et al., 1988]. Whether a specification is to be viewed as fixed or as random should be decided by asking what would happen if the benefitgenerating structure presumed at one site were transferred to another. It should not be decided by the arbitrary availability and the habitual use of a particular formulation of the data-generating process such as classical least squares.

Think of the distribution of a random coefficient as a prior distribution. This line of reasoning leads naturally to Bayesian or empirical Bayes approaches to estimation. Rubin [1980], Kuczera [1982], DuMouchel and Harris [1983], Aigner and Leamer [1984], Chamberlain [1988], and others have convincingly demonstrated that empirical Bayes estimators are statistically more efficient than classical least squares using individual samples. This increase in efficiency occurs because the site-specific application of empirical Bayes estimators avoids the duplication of information that already exists elsewhere.

What distinguishes the empirical Bayes approach from its classical standard linear counterpart is the presence of a prior distribution for the $\beta$ vector, the vector of parameters that influence benefits. Given that the results or the data of previous benefit studies are exchangeable and can be stated in conformable units, the prior can be gleaned from these studies. Specifically, one defines (3), $\beta \sim N\left(\mu, \sigma_{\beta}^{2}\right)$, such that $\mu$ is the mean vector across sites of a $\beta$ and $\sigma_{\beta}^{2}$ is the variance-covariance matrix.

Empirical Bayes techniques may appear convoluted, but they in fact have a simple rationale. Consider a set of existing site-specific benefit studies that have been useful for policymakers. Suppose that limited research resources do 
not allow a benefit assessment to be done at a particular site but that governmental mandates require a benefit magnitude be assigned to a proposed pollution control activity at the site. Often, the missing benefit number would be replaced by the average value of past studies. This average value is thus considered an estimator of the benefits at the new site, as would a fresh assessment of the new site had it been produced. Suppose now that the fresh assessment has been produced. In this case, either the fresh assessment or the average value from past studies can be used to inform the pollution control decision. The empirical Bayes approach says a weighted average of the two estimates is better than either alone.

Following Morris [1983], Leamer [1978], and Zellner [1986], assume we have benefit estimates for $i=1, \cdots, n$ sites. Let $y_{i}$ be the average benefit estimate across the $i$ sites and let $h_{i}$ be the benefit at the $n+1$ site. Assume

$$
\left(y_{i} \mid h_{i}\right) \sim N\left(h_{i}\left(\sigma_{\beta}^{2}\right)\right) \text {; }
$$

that is, given the benefit at the $n+1$ site, the benefit estimates at other sites are distributed normally with variance $\sigma_{\beta}^{2}$ about the $n+1$ site benefit. Efron and Morris [1975] show that the normality assumption is not essential to the empirical Bayes approach. Further assume

$$
\left(h_{i} \mid \mu, \sigma_{\beta}^{2}\right)=N\left(z_{i}^{\prime} \beta, \sigma_{\beta}^{2}\right)
$$

The parameters of interest, the parameters that determine benefits at the $n+1$ site, can be estimated by either $\mu$ or $z_{i} \hat{\beta}$, where $\hat{\beta}$ is an estimator of $\beta$. A compromise estimator that incorporates both of these estimators with efficiency better than either estimator alone is

$$
\beta_{i}^{*}=\left(z_{i}^{\prime} z_{i}+\lambda_{i} I\right)^{-1}\left(z_{i}^{\prime} z_{i} \hat{\beta}_{i}+\lambda_{i} \mu\right),
$$

where $I$ is the identify matrix. The information from previous studies is combined with the sample data for the $n+1$ site. The updated result of this combination is a matrixweighted average of the information contained in $\mu$ with the sample information for the $n+1$ site. The $\lambda_{i}$ parameter $(0$ $\left.\leq \lambda^{i} \leq \infty\right)$ dictates the relative weight devoted to each information type. These weights can be viewed similarly to those in (4). They represent the policymaker's or her agent's belief in the extent to which one can transfer the results of previous benefits studies to the $n+1$ site.

Referring again to (1) and (3), if the parameters $\sigma_{\beta}^{2}, \sigma_{\gamma}^{2}, \sigma_{\alpha}^{2}$, and $\mu$ were known and if $\sigma_{\gamma}^{2}$ was the same at every site, then the optimal estimator could be obtained from (14). But these statistics will usually be unknown, and even if they are known the $\sigma_{\gamma}^{2}$ will typically be unequal across sites. When the $\sigma_{\gamma}^{2}$ are equal, the EM algorithm of Dempster et al. [1977] is available to obtain closed form, maximum likelihood estimates of the $\beta$. This accomplishes little, however, if the variances are unknown either absolutely or, more significantly according to (8)-(10), relative to each other. The empirical Bayes procedures overcome this awkward feature by calculating point estimates of these dispersion parameters and then substituting them into the dispersion expressions presented by Morris [1983] and elsewhere in order to make inferences about the $\beta$. Typically, these dispersions are estimated by maximum likelihood so that they are asymptotically efficient. It follows then that all empirical Bayes inferences about $\beta$ are conditional on point estimates of the dispersions.
The extra effort required to resolve issues of unequal or unknown dispersions in order to gain the increased efficiencies of empirical Bayes techniques will serve little purpose if there is no efficiency to be gained. Otherwise, one might just as well enjoy the comforts of uncompromised fixed coefficient estimators such as classical least squares. Empirical Bayes takes the common benefit that exchangeability implies as a maintained hypothesis. If it is absent or small, observations 1 and 2 and expression (14) suggest that Bayesian approaches be set aside. In order to make this decision, one obviously must know whether $\sigma_{\beta}^{2}$ is large or small. In the next section, we propose an empirical Bayes diagnostic for assessing the size of $\sigma_{\beta}^{2}$. We also apply this diagnostic in the context of a hedonic study of pollution control benefits. The power of the diagnostic arises from its ability to identify those sources of parameter variability most likely to discourage extrapolations from prior studies.

\section{CONDITIONS FOR A COMMON BENEFIT}

In this section, we evaluate the stochastic restrictions that must be imposed on a site-specific hedonic price function in order to shrink its parameters toward the estimates arising from an empirical Bayes treatment that pools estimates from a number of sites. The severity of the required restrictions provides a measure of the degree of commonality that must be present in order to extrapolate results from previous hedonic studies to the site now being studied. The $\lambda$ parameter in (14) is the key to this diagnosis. The parameter allows policymakers to evaluate the place of their ongoing and their proposed benefits studies in the ensemble of existing studies.

\section{Technique}

Chamberlain and Leamer [1976] and Leamer [1982] argue that a Bayesian analysis based on a single prior distribution will attract little respect because such distributions are difficult to measure without error or because of seemingly reasonable differences in the prior judgments of policymakers and their agents. So as to give credence to the range of plausible priors and to establish those features of a prior having major influences on a posterior, they take the prior mean, $\mu$, as given and develop the correspondence between transforms of the prior precision, $\left(\sigma_{\beta}^{2}\right)^{-1}$, and the posterior mean. Leamer [1982] has shown how restrictions on $\sigma_{\beta}^{2}$ imply restrictions on the feasible set of posterior means. The $\sigma_{\beta}^{2}$ matrix is allowed to assume any value in the interval $\lambda^{-\infty} \hat{\sigma}_{\beta}^{2} \leq \sigma_{\beta}^{2} \leq \lambda^{\infty} \bar{\sigma}_{\beta}^{2}$, where $\hat{\sigma}_{\beta}^{2}$ and $\bar{\sigma}_{\beta}^{2}$ are respectively lower and upper bounds, where $\sigma_{\beta}^{2}-\hat{\sigma}_{\beta}^{2}$ and $\bar{\sigma}_{\beta}^{2}-\sigma_{\beta}^{2}$ are positive semidefinite, and where $\lambda$ is any nonnegative constant scaler.

We use Leamer's [1982] bounded variance prior technique to obtain root-mean-square error (RMSE) forecasts and to compute coefficient extreme bounds in a hedonic property price expression. Using the technique, we evaluate the contribution made by the priors to accurate predictions of property price levels. With the extreme bounds, we evaluate the sensitivity of individual parameters to a weakening of the precision of our priors. If use of these prior means, weighted by different prior precision matrices, reduces the RMSE and the extreme bounds of our posterior estimates, we conclude that sample means from other locales and times are consistent with our data, that is, are equivalent to a large random sample from our data set. Exchangeability would then be 
strong and a unified hedonic market can be said to exist, given our prior information and our data. However, if differentially weighted prior means substantially increase the RMSE or the extreme bounds of our posterior estimates, we conclude that sample means from other locales and times are not consistent with our data, that exchangeability is weak, and that multiple hedonic markets exist.

\section{Data}

Our data set embodies information on more than 297 structural and neighborhood attributes for 1283 Federal Housing Administration (FHA) insured, detached, singlefamily residential properties in Chicago, from 1964 through 1967. These properties constitute nearly half of such properties sold in the city during the 4-year period. In spite of the peculiar features of FHA-insured financing, the sale prices of the homes in our sample are comparable to the assessed values and owner-reported values of homes in their immediate neighborhoods. All three measures had 1965 mean values of about $\$ 16,000$, with standard deviations between $\$ 3000$ and $\$ 3700$. Sale prices of the FHA-insured sample homes range up to $\$ 60,000$. Strictly cash transactions are excluded.

Investigator energies and analytical tractability, if not computer limitations, require that parameters be economized in any empirical exercise. In a survey of 15 published hedonic property price studies, Atkinson and Crocker [1987] found that regression coefficients for a total of 110 distinct covariates were reported. Even though our data set contains some measure of nearly all of these covariates, the construction of a composite model would make us and, we presume, most readers uncomfortable. We instead confine our attention to a parsimonious vector of 11 covariates which have very frequently appeared in the published literature, are important contributors in terms of explanatory power to property price, and are representative of a range of property structure and neighborhood attributes. One could easily incorporate additional variables measuring proximity to water recreation sites or other water-related attributes. We adopt a semilog specification of a reduced form hedonic price equation with the aforementioned 11 covariates as the only relevant explanatory variables. Table 1 displays the definitions, sources, sample and prior means, standard deviations, and places and times of sales price for the 11 covariates. At least for the Chicago data set, additional covariates add very little explanatory power and, as shown by Atkinson and Crocker [1987], substantially increase the likelihood of confounded interpretations arising from measurement error problems.

\section{Prior Means}

Table 1 presents our prior means, assuming that a semilog form is appropriate for the hedonic price expression. With three exceptions, after having accounted for differences among studies in units of measurement, each prior mean is the median estimated coefficient among the aforementioned 15 hedonic studies. Since there is little agreement in this literature about what might constitute "informative" prior means for air pollution, time of sale, and property taxes, we treat these three covariates as "free" and set their prior means equal to their respective posterior means calculated in a preliminary estimate in which diffuse priors were assumed for all coefficients. The prior variance for each of the three free variables is set at zero, since in specifying a variable as free, we implicitly assume that the posterior variance of its coefficient is completely described by the sample data. The prior mean for the constant is calculated by minimizing, subject to the prior restrictions, the sum of the squared errors. We view the remaining eight covariates as doubtful in the sense that we doubt that their coefficients differ from their prior values. However, we allow the prior variance of these doubtful variables to be described solely by the sample variance and to lie in an interval $\lambda^{-1} \hat{\sigma}_{\beta}^{2} \leq \sigma_{\beta}^{2} \leq \lambda^{1} \bar{\sigma}_{\beta}^{2}$. By varying $\lambda$ over a broad range, we can entertain a wide variety of prior opinions about the contribution of particular property attributes to selling price. Wider bounds for our posterior estimates imply less transferability of the results of previous studies to the locale and the time of interest.

In Table 2, the column for $\lambda=1$ shows the initial Bayesian estimates with 320 observations. The estimates are "initial" because $\lambda=1$ means that the initially input prior covariance matrix is employed. As one proceeds rightward in the table, the range of the prior covariance matrix becomes progressively larger. For example, if $\lambda=4$, the range of prior standard errors can be twice as large or half as small as that in $\sigma_{\beta}^{2}$. At $\lambda=\infty$, this range becomes unbounded. The extreme bounds, $U$ and $L$, represent the upper and the lower bounds of all posterior mean estimates for a given $\lambda$.

In general, Table 2 shows that unless there is a broad consensus that the prior covariance matrix is very nearly exact as initially input, the ranges of the estimates for all covariates except living area, time of sale, and property taxes typically include the origin. Zero is much closer to the midpoint of the bounds of the neighborhood attributes such as percent black and air pollution than it is for structural attributes of the residence. A similar pattern emerges with sample sizes of 80 and 160 , though increasing the sample size to 320 does cause considerable compressions in bounds. Generally, but especially for neighborhood attributes, minor differences in $\lambda$ lead to large differences in posterior estimates.

The contribution to a narrowing of the bounds of a doubling of sampling size is consistently greater than the contribution which results from compressing the prior covariance matrices. For example, if $\lambda=16$, an increase from 80 to 160 observations reduces the bounded interval for the coefficient of air pollution from $20.55 \times 10^{-3}$ to $7.70 \times 10^{-3}$, a reduction of $63 \%$. However, if $\lambda$ is set equal to 8 rather than 16 for 80 observations, such that the precision of one's prior information is increased by a factor of 2 , the bounded interval for the coefficient of air pollution declines by only $38 \%$. The bounded intervals for other covariates behave similarly. We therefore conclude that when sample size is rather small a doubling in the number of observations from the sample locale and time will contribute substantially more to the precision of posterior estimates than will a twofold increase in the precision of one's prior information drawn from the existing hedonic property value literature. Again, if $\lambda=16$, an increase from 160 to 320 observations decreases the bounded interval for the coefficient of air pollution from $7.70 \times 10^{-3}$ to $4.05 \times 10^{-3}$, a reduction of $47 \%$ in interval width. However, if $\lambda$ is set equal to 8 rather than 16 for 160 observations, the bounded interval for the coefficient of age declines by only $30 \%$. Thus, although prior information drawn from other times and places does contribute in 
TABLE 1. Variable Definitions, Sources, and Summary Statistics

\begin{tabular}{|c|c|c|c|c|c|c|c|}
\hline Covariate & Definition and Source & $\begin{array}{l}\text { Sample } \\
\text { Mean }\end{array}$ & $\begin{array}{l}\text { Sample } \\
\text { Standard } \\
\text { Deviation }\end{array}$ & Prior Mean & Source of Prior & $\begin{array}{l}\text { Locale of } \\
\text { Prior }\end{array}$ & $\begin{array}{l}\text { Year(s) of } \\
\text { Prior }\end{array}$ \\
\hline Price & dollars; source, FHA. & 16,228 & $\begin{array}{l}\text { Dependent } \\
3,620\end{array}$ & & & & \\
\hline Age & age in years; source, FHA. & 31 & $\begin{array}{c}\text { Exogenous } \\
18\end{array}$ & $-2.50 \times 10^{-3}$ & $\begin{array}{l}\text { Schnare and } \\
\text { Struyk }[1976]\end{array}$ & Boston & 1971 \\
\hline Lot size & square feet; source, FHA. & 4,298 & 2,348 & $3.00 \times 10^{-6}$ & $\begin{array}{l}\text { Schnare and } \\
\text { Struyk }[1976]\end{array}$ & Boston & 1971 \\
\hline Living area & square feet; source, FHA. & 1,216 & 309 & $3.00 \times 10^{-4}$ & $\begin{array}{l}\text { Johnson and } \\
\text { Lea }[1982]\end{array}$ & Buffalo & 1978 \\
\hline Crime & $\begin{array}{l}\text { the mean } 1962-1965 \text { community } \\
\text { area male delinquency rate as a } \\
\text { percentage of the rate for the } \\
\text { Chicago metropolitan area; } \\
\text { source, Shaw and } M \text { M } \text { ay }[1968] \text {. }\end{array}$ & 0.80 & 0.44 & $-5.40 \times 10^{-2}$ & $\begin{array}{l}\text { Harrison and } \\
\quad \text { Rubinfeld } \\
{[1978]}\end{array}$ & Boston & 1970 \\
\hline Distance & $\begin{array}{l}\text { distance in tenths of miles to the } \\
\text { intersection of State and } \\
\text { Madison Streets in Loop area of } \\
\text { downtown Chicago; source: } \\
\text { contemporary city maps. }\end{array}$ & 100 & 22 & $-2.18 \times 10^{-3}$ & Butler [1982] & St. Louis & 1967 \\
\hline $\begin{array}{l}\text { Median } \\
\text { income }\end{array}$ & $\begin{array}{l}\text { median } 1960 \text { household income in } \\
\text { dollars of the census tract in } \\
\text { which the residence was located; } \\
\text { source, U.S. Bureau of the } \\
\text { Census [1962, Table P-1]. }\end{array}$ & 7,549 & 1,120 & $2.80 \times 10^{-5}$ & $\begin{array}{l}\text { Jud and Watts } \\
\text { [1981] }\end{array}$ & Charlotte & 1977 \\
\hline $\begin{array}{r}\text { Percent } \\
\text { black }\end{array}$ & $\begin{array}{l}\text { percentage black of April } 1966 \\
\text { population in the community } \\
\text { area; source, DeVise [1967]. }\end{array}$ & 0.13 & 0.28 & $-2.70 \times 10^{-2}$ & $\begin{array}{l}\text { Brookshire et } \\
\quad \text { al. [1982] }\end{array}$ & Los Angeles & $1977-1978$ \\
\hline $\begin{array}{l}\text { School } \\
\text { quality }\end{array}$ & $\begin{array}{l}\text { arithmetic sum }(0-10) \text { by school } \\
\text { district of mean } 1963 \text { reading and } \\
\text { arithmetic achievement tests in } \\
\text { public elementary schools; } \\
\text { source, Havighurst [1964, p. 39]. }\end{array}$ & 6.68 & 0.05 & $5.60 \times 10^{-2}$ & Butler [1982] & St. Louis & 1967 \\
\hline $\begin{array}{l}\text { Air } \\
\text { pollution }\end{array}$ & $\begin{array}{l}\text { annual arithmetic mean monthly } \\
\text { total suspended participation } \\
\left(\mu \mathrm{g} / \mathrm{m}^{3}\right) \text { for } 48 \text {-month period } \\
\text { from Jan. } 1964 \text { to Jan. 1968; } \\
\text { source: Chicago Air Pollution } \\
\text { Control District records. }\end{array}$ & 130.15 & 11.24 & diffuse $^{a}$ & sample & Chicago & $1964-1967$ \\
\hline & $\begin{array}{l}\text { month of sale: Jan. } 1964=1, \cdots, \\
\text { Dec. } 1967=48 .\end{array}$ & 26.37 & 13.52 & diffuse $^{a}$ & sample & Chicago & 1964-1967 \\
\hline $\begin{array}{c}\text { Property } \\
\text { taxes }\end{array}$ & $\begin{array}{l}\text { annual property taxes in tens of } \\
\text { dollars and any continuing } \\
\text { nonrepayable special } \\
\text { assessments; source, FHA. }\end{array}$ & 26.05 & 8.08 & diffuse $^{a}$ & sample & Chicago & $1964-1967$ \\
\hline
\end{tabular}

1 square foot equals $0.0929 \mathrm{~m}^{2} ; 1$ mile $=1.609 \mathrm{~km}$.

${ }^{a}$ The prior means are computed via restricted least squares by minimizing the sum of squared errors subject to the prior means for all other coefficients imposed as restrictions.

absolute terms as a supplement to sample information, it contributes only modestly relative to additional sample information.

The findings reported in Table 3 reinforce the above results. This table shows the minimum prior variance intervals, stated in terms of joint values of the scalars, $\lambda^{-1}$ and $\lambda$, which will cause the corresponding intervals for the posterior means to pass through the origin. For eight of the 11 covariates, the intervals get broader, sometimes much broader, as sample size increases, implying that a wider range of prior opinions about coefficients can be accommodated without causing coefficient sign switches. However, the bounds for the neighborhood covariates which are often the focus of research interest remain quite narrow. Consequently, given our prior information and our data, seemingly minor differences in prior opinions about the influences of these covariates upon sales prices can lead to major discrepancies in estimated posteriors. Thus our pooling of data from different places and times generated substantial variation in the hedonic prices of neighborhood attributes but much less variation in the hedonic prices for structural residence attributes. In our illustration, it thus follows that the hedonic prices of neighborhood attributes were generated in multiple housing markets, while the hedonic prices of structural attributes originated in a unified market. We therefore conclude that prior information about the contributions of neighborhood attributes to property prices is not readily exchanged with the time and locale of our Chicago data. However, prior information on the contribution of structural attributes is readily exchanged. 
TABLE 2. Bounded Variance Prior Estimates With 320 Observations

\begin{tabular}{|c|c|c|c|c|}
\hline Covariate & $\lambda=1$ & $\lambda=4$ & $\lambda=16$ & $\lambda=\infty$ \\
\hline \multicolumn{5}{|l|}{ Age } \\
\hline U & $-2.95 \times 10^{-3}$ & $-1.51 \times 10^{-3}$ & $0.98 \times 10^{3}$ & $1.54 \times 10^{-3}$ \\
\hline $\mathbf{L}$ & $-2.95 \times 10^{-3}$ & $-4.39 \times 10^{-3}$ & $-6.73 \times 10^{-3}$ & $-7.24 \times 10^{-3}$ \\
\hline \multicolumn{5}{|l|}{ Lot size } \\
\hline $\mathbf{U}$ & $1.46 \times 10^{-5}$ & $1.92 \times 10^{-5}$ & $2.79 \times 10^{-5}$ & $3.00 \times 10^{-5}$ \\
\hline $\mathbf{L}$ & $1.46 \times 10^{-5}$ & $0.77 \times 10^{-5}$ & $-0.79 \times 10^{-5}$ & $-1.18 \times 10^{-5}$ \\
\hline \multicolumn{5}{|l|}{ Living area } \\
\hline $\mathbf{U}$ & $1.61 \times 10^{-4}$ & $2.31 \times 10^{-4}$ & $3.82 \times 10^{-4}$ & $4.20 \times 10^{-4}$ \\
\hline $\mathbf{L}$ & $1.61 \times 10^{-4}$ & $1.14 \times 10^{-4}$ & $0.40 \times 10^{-4}$ & $0.24 \times 10^{-4}$ \\
\hline \multicolumn{5}{|l|}{ Crime } \\
\hline $\mathbf{U}$ & $1.51 \times 10^{-2}$ & $5.23 \times 10^{-2}$ & $1.15 \times 10^{-1}$ & $1.30 \times 10^{-1}$ \\
\hline $\mathbf{L}$ & $1.51 \times 10^{-2}$ & $-6.52 \times 10^{-2}$ & $-1.93 \times 10^{-1}$ & $-2.22 \times 10^{-1}$ \\
\hline \multicolumn{5}{|l|}{ Distance } \\
\hline U & $-2.18 \times 10^{-4}$ & $3.95 \times 10^{-4}$ & $1.47 \times 10^{-3}$ & $1.174 \times 10^{-3}$ \\
\hline $\mathbf{L}$ & $-2.18 \times 10^{-4}$ & $-1.68 \times 10^{-3}$ & $-4.26 \times 10^{-3}$ & $-4.86 \times 10^{-3}$ \\
\hline \multicolumn{5}{|c|}{ Median income } \\
\hline $\mathbf{U}$ & $2.07 \times 10^{-5}$ & $3.75 \times 10^{-5}$ & $6.88 \times 10^{-5}$ & $7.64 \times 10^{-5}$ \\
\hline $\mathbf{L}$ & $2.07 \times 10^{-5}$ & $-0.36 \times 10^{-5}$ & $-3.79 \times 10^{-5}$ & $-4.54 \times 10^{-5}$ \\
\hline \multicolumn{5}{|l|}{ Percent black } \\
\hline U & $1.00 \times 10^{-1}$ & $1.43 \times 10^{-1}$ & $2.25 \times 10^{-1}$ & $2.46 \times 10^{-1}$ \\
\hline $\bar{L}$ & $1.00 \times 10^{-1}$ & $0.17 \times 10^{-1}$ & $-1.53 \times 10^{-1}$ & $-1.95 \times 10^{-1}$ \\
\hline \multicolumn{5}{|l|}{ School quality } \\
\hline $\mathbf{U}$ & $3.43 \times 10^{-2}$ & $6.68 \times 10^{-2}$ & $1.22 \times 10^{-1}$ & $1.35 \times 10^{-1}$ \\
\hline $\mathbf{L}$ & $3.43 \times 10^{-2}$ & $-1.82 \times 10^{-2}$ & $-0.86 \times 10^{-1}$ & $-0.93 \times 10^{-1}$ \\
\hline \multicolumn{5}{|l|}{ Air pollution } \\
\hline U & $-1.48 \times 10^{-3}$ & $-0.83 \times 10^{-3}$ & $0.33 \times 10^{-3}$ & $0.60 \times 10^{-3}$ \\
\hline $\mathbf{L}$ & $-1.48 \times 10^{-3}$ & $-2.28 \times 10^{-3}$ & $-3.72 \times 10^{-3}$ & $-4.05 \times 10^{-3}$ \\
\hline \multicolumn{5}{|l|}{ Time of sale } \\
\hline $\mathbf{U}$ & $2.26 \times 10^{-3}$ & $2.64 \times 10^{-3}$ & $3.40 \times 10^{-3}$ & $3.59 \times 10^{-3}$ \\
\hline $\mathbf{L}$ & $2.26 \times 10^{-3}$ & $1.97 \times 10^{-3}$ & $1.43 \times 10^{-3}$ & $1.29 \times 10^{-3}$ \\
\hline \multicolumn{5}{|c|}{ Property taxes } \\
\hline U & $1.38 \times 10^{-2}$ & $1.65 \times 10^{-2}$ & $2.09 \times 10^{-2}$ & $2.11 \times 10^{-2}$ \\
\hline L & $1.38 \times 10^{-2}$ & $1.17 \times 10^{-2}$ & $0.77 \times 10^{-2}$ & $0.75 \times 10^{-2}$ \\
\hline Constant & 8.85 & 8.85 & 8.85 & \\
\hline
\end{tabular}

Here, $\lambda^{-1} V_{0} \leq V \leq \lambda V_{0}$. U denotes upper extreme bound; $L$, lower extreme bound.

\section{Summary and Conclusions}

In our theoretical development, we show that the value of transferring benefit estimates among sites or across time depends upon the variance of common benefit estimates relative to the variance of site-specific estimates. Sites having benefits in common are exchangeable because they share a common structure of benefit generation. Given that the distribution of a random coefficient can be viewed as a prior distribution, we propose the use of a random coefficient estimator known as empirical Bayes to exploit the research efficiencies that exchangeability makes possible. When the empirical Bayes applied to a specific site pulls or shrinks its estimate toward the mean estimate for other sites, policymakers have a more reliable standard with which to assess site-specific estimates. The degree of shrinkage achieved depends on the variances of the parameters that define the benefit structure as well as the sampling variance. However, a parameter variance often cannot be precisely measured. There are therefore grounds for exploring the consequences of a plausible range of parameter variances. In addition, as Marin [1986] and others have shown, the shrinkage procedure may be sensitive to the exchangeability assumption. For example, if parameter estimates vary across sites as a function of ignored site attributes, the shrinkage will bias estimates because noninformative priors are being employed.
In order to provide an example of an exploration of the mix of priors or regions of the parameter space where meaningful increases in research efficiency can be achieved, we applied Leamer's [1982] bounded variance prior technique to a hedonic property value data set. This technique allows one to assess the impact that alternative restrictions upon the prior variances will have upon the feasible set of posterior means. Our application illustrated the important point that when samples are small, shrinkage toward the prior mean is most pronounced, and most desired, to compensate for the instabilities of conventional estimates. As sample size increased the shrinkage estimates converged to least squares estimates. We found for our data set that the research efficiency gains from increased sample size generally dominate the gains from shrinkage, implying that policymakers would have little incentive to extrapolate the hedonic benefits estimates from previous studies to new sites. Nevertheless, we also showed that only weak restrictions upon prior variances were required to justify extrapolation of structural residence parameter estimates in hedonic property value studies to new sites and times. In conclusion, our theoretical framework demonstrates the advisability of researchers' and research administrators' considering how their proposed and ongoing studies fit into the matrix of existing studies. Our empirical results provide an example of how they might accomplish this. 
TABLE 3. Smallest Joint Values of $\lambda$ and $\lambda^{-1}$ Leading to Coefficient Sign Switches

\begin{tabular}{|c|c|c|c|}
\hline \multirow[b]{2}{*}{ Covariate } & \multicolumn{3}{|c|}{ Number of Observations } \\
\hline & 80 & 160 & 320 \\
\hline \multicolumn{4}{|l|}{ Age } \\
\hline$\lambda$ & 4.00 & 8.00 & 16.00 \\
\hline$\lambda^{-1}$ & 0.25 & 0.13 & 0.16 \\
\hline \multicolumn{4}{|l|}{ Lot size } \\
\hline$\lambda$ & 16.00 & 16.00 & 8.00 \\
\hline$\lambda^{-1}$ & 0.06 & 0.06 & 0.13 \\
\hline \multicolumn{4}{|l|}{ Living area } \\
\hline$\lambda$ & 4.00 & 8.00 & $\infty$ \\
\hline$\lambda^{-1}$ & 0.25 & 0.13 & $-\infty$ \\
\hline \multicolumn{4}{|l|}{ Crime } \\
\hline$\lambda$ & 2.00 & 4.00 & 2.00 \\
\hline$\lambda^{-1}$ & 0.50 & 0.25 & 0.50 \\
\hline \multicolumn{4}{|l|}{ Distance } \\
\hline$\lambda$ & 4.00 & 2.00 & 2.00 \\
\hline$\lambda^{-1}$ & 0.25 & 0.50 & 0.50 \\
\hline \multicolumn{4}{|c|}{ Median income } \\
\hline$\lambda$ & 2.00 & 4.00 & 4.00 \\
\hline$\lambda^{-1}$ & 0.50 & 0.25 & 0.25 \\
\hline \multicolumn{4}{|l|}{ Percent black } \\
\hline$\lambda$ & 2.00 & 4.00 & 8.00 \\
\hline$\lambda^{-1}$ & 0.50 & 0.25 & 0.13 \\
\hline \multicolumn{4}{|c|}{ School quality } \\
\hline$\lambda$ & 2.00 & 2.00 & 4.00 \\
\hline$\lambda^{-1}$ & 0.50 & 0.50 & 0.25 \\
\hline \multicolumn{4}{|l|}{ Air pollution } \\
\hline$\lambda$ & 2.00 & 4.00 & 16.00 \\
\hline$\lambda^{-1}$ & 0.50 & 0.25 & 0.06 \\
\hline \multicolumn{4}{|l|}{ Time of sale } \\
\hline & 8.00 & $\infty$ & $\infty$ \\
\hline$\lambda^{-1}$ & 0.13 & $-\infty$ & $-\infty$ \\
\hline \multicolumn{4}{|c|}{ Property taxes } \\
\hline$\lambda$ & $\infty$ & $\infty$ & $\infty$ \\
\hline$\lambda^{-1}$ & $-\infty$ & $-\infty$ & $-\infty$ \\
\hline
\end{tabular}

\section{REFERENCES}

Adams, R. M., T. D. Crocker, and R. W. Katz, Assessing the adequacy of natural science information: A Bayesian approach, Rev. Econ. Stat., 66, 568-575, 1984.

Aigner, D. J., and E. E. Leamer, Estimation of time-of-use pricing response in the absence of experimental data, $J$. Econometrics, 26, 205-227, 1984.

Atkinson, S. E., and T. D. Crocker, A Bayesian approach to assessing the robustness of hedonic property value studies, $J$. Appl. Econometrics, 2, 27-45, 1987.

Brookshire, D. S., M. A. Thayer, W. D. Schulze, and R. C. d'Arge, Valuing public goods: A comparison of survey and hedonic approaches, Am. Econ. Rev., 72, 165-176, 1982.

Butler, R. V., The specification of hedonic indices for urban housing, Land Econ., 58, 96-108, 1982.

Chamberlain, G., and E. E. Leamer, Matrix weighted averaged and posterior bounds, J. R. Stat. Soc., Ser. B, 37, 73-84, 1976.

Chamberlain, P. J., An empirical Bayes approach to modeling drought, West. J. Agric. Econ., 13, 92-99, 1988.

Chow, G. C., A comparison of the information and the posterior probability criteria for model selection, J. Econometrics, 16, 21-33, 1981

Crocker, T. D., Benefit-cost analysis of benefit-cost analyses, in Cost Benefit Analysis and Water Pollution Policy, edited by H. M. Peskin and E. P. Seskin, pp. 341-360, Urban Institute, Washington, D. C., 1975.

de Finetti, B., Induction, Probability and Statistics, John Wiley, New York, 1972.

Dempster, A. P., N. M. Laird, and D. B. Rubin, Maximum likelihood from incomplete data via the EM algorithm (with discussion), J. R. Stat. Soc., Ser. B, 39, 1-38, 1977.
DeVise, P., Chicago's widening color gap, Rep. 2, 98 pp., Interuniv. Soc. Res. Comm., Chicago, III., Dec. 1967.

DuMouchel, W. H., and J. E. Harris, Bayes methods for combining the results of cancer studies in humans and other species, J. Am. Stat. Assoc., 78, 293-308, 1983.

Efron, B., and C. Morris, Data analysis using Stein's estimator and its generalizations, J. Am. Stat. Assoc, 70, 311-319, 1975.

Harrison, D., Jr., and D. L. Rubinfeld, Hedonic housing prices and the demand for clean air, J. Environ. Econ. Manage., 5, 81-102, 1978.

Havighurst, R. J., The Public Schools of Chicago, 224 pp., Board of Education of the City of Chicago, Chicago, Ill., 1964.

Johnson, M. S., and M. J. Lea, Differential capitalization of local public service characteristics, Land Econ., 58, 189-203, 1982.

Jud, G. D., and J. M. Watts, Schools and housing values, Land Econ., 57, 459-470, 1981.

Judge, G. G., R. C. Hill, W. E. Griffiths, H. Lutkepohl, and T. Lee, Introduction to the Theory and Practice of Econometrics, John Wiley, New York, 1988.

Just, R. E., D. L. Hueth, and A. Schmitz, Applied Welfare Economics and Public Policy, Prentice-Hall, Englewood Cliffs, N. J., 1982.

Klein, R. W., L. C. Rafsky, D. S. Sibley, and R. D. Willig, Decisions with estimation uncertainty, Econometrica, 46, 1363$1387,1978$.

Kuczera, G., Combining site-specific and regional information: An empirical Bayes approach, Water Resour. Res., 18, 306-314, 1982.

Leamer, E. E., Specification Searches: Ad Hoc Inference With Nonexperimental Data, John Wiley, New York, 1978.

Leamer, E. E., Sets of posterior means with bounded priors, Econometrica, 50, 725-736, 1982.

Lindley, D. V., and A. F. M. Smith, Bayes estimates for the linear model, J. R. Stat. Soc., Ser. B, 34, 1-41, 1972.

Marin, C. M., Parameter estimation in water resources planning and management, Water Resour. Res., 22, 353-360, 1986.

Morris, C. N., Parametric empirical Bayes inference: Theory and applications (with discussion), J. Am. Stat. Assoc., 78, 47-65, 1983.

Rubin, D. B., Using empirical Bayes techniques in the law school validity studies, J. Am. Stat. Assoc., 75, 1325-1333, 1980.

Schnare, A. B., and R. J. Struyk, Segmentation in urban housing markets, J. Urban Econ., 3, 146-166, 1976.

Shaw, C. R., and H. D. McKay, Juvenile Delinquency and Urban Areas, 338 pp., University of Chicago Press, Chicago, III., 1968.

Smith, V. K., and Y. Kaoru, Signals or noise? Explaining the variation in recreation benefit estimates, Am. J. Agric. Econ., 72, $419-433,1990$.

Swamy, P. A. V. B., Efficient inference in a random coefficient regression model, Econometrica, 38, 311-323, 1970.

Swamy, P. A. V. B., R. K. Conway, and M. R. LeBlanc, The stochastic coefficients approach to econometric modeling, I, A critique of fixed coefficients models, J. Agric. Econ. Res., 40, $2-10,1988$.

U.S. Bureau of the Census, 1960 Census of Population: Chicago, U.S. Government Printing Office, Washington, D. C., 1962.

Walsh, R. G., D. M. Johnson, and J. R. McKean, Issues in nonmarket evaluation and policy application: $A$ retrospective glance, West. J. Agric. Econ., 14, 178-188, 1989.

Zellner, A., On assessing prior distributions and Bayesian regression analysis with $g$-prior distributions, in Bayesian Inference and Decision Techniques: Essays in Honor of Bruno de Finetti, edited by P. K. Goet and A. Zellner, pp. 233-243, North-Holland, Amsterdam, 1986.

S. E. Atkinson, Department of Economics, University of Georgia, Athens, GA 30601.

T. D. Crocker, Department of Economics and Finance, University of Wyoming, Laramie, WY 82071.

J. F. Shogren, Department of Economics, Iowa State University, Ames, IA 50011.

(Received November 14, 1990; revised February 15, 1991; accepted January 22, 1991.) 